\title{
Improved Tomlinson-Harashima Precoding for Ultra Reliable Communication in Intelligent Transportation Systems
}

\author{
Shibiao He $\mathbb{D},{ }^{1}$ Xinyi Yang $\mathbb{D}^{2},{ }^{2}$ and Yong Liao $\mathbb{D}^{2}$ \\ ${ }^{1}$ School of Electronic Information, Chongqing Institute of Engineering, Chongqing 400056, China \\ ${ }^{2}$ School of Microelectronics and Communication Engineering, Chongqing University, Chongqing 400044, China \\ Correspondence should be addressed to Shibiao He; hdoctor@vip.sina.com
}

Received 23 February 2021; Revised 8 March 2021; Accepted 24 March 2021; Published 5 April 2021

Academic Editor: Renke Han

Copyright (c) 2021 Shibiao He et al. This is an open access article distributed under the Creative Commons Attribution License, which permits unrestricted use, distribution, and reproduction in any medium, provided the original work is properly cited.

Cyber-physical systems (CPSs) are characterized by integrating computation and physical processes. To cope with the challenges of the application of the CPSs in all kinds of environments, especially the cellular vehicle-to-everything (C-V2X) which needs high quality end-to-end communication, the robustness and reliability for CPSs are very crucial. Aiming at the technical challenges of information transmission caused by the fading effect and the fast time-varying characteristics of the channel for C-V2X communication, an improved Tomlinson-Harashima precoding (THP) algorithm for multiple input multiple output (MIMO) systems is proposed. Channel state information (CSI) and correlation are exploited to compensate instantaneous CSI, which could reflect current real-time channel status exactly. Further, the iterative water filling power allocation algorithm and the multiuser scheduling algorithm based on the greedy algorithm are jointly optimized and applied to the THP, which could improve the system performance. Simulation results show that the proposed algorithm can be efficiently applied to high-speed mobility scenarios and improve bit error ratio (BER) performance as well as spectrum utilization.

\section{Introduction}

In recent years, the construction of global smart city is accelerated, which makes cyber-physical systems (CPSs) develop rapidly [1]. Large-scale networked CPSs are deployed to address global needs in areas such as energy $[2,3]$, transportation $[4,5]$, power system $[6,7]$, and autocontrol $[8,9]$. As an intelligent system integrating computing, communication, and control, any problems may affect the normal operation of the CPSs. Thus, the stability of CPSs which includes system reliability and robustness should be considered and improved. CPSs should respond to the input of the system timely and effectively, especially for cellular vehicle-to-everything (C-V2X) communication scenarios. The vehicle in the $\mathrm{C}-\mathrm{V} 2 \mathrm{X}$ adopts short distance wireless communication technology to communicate between vehicle-to-vehicle (V2V) and vehicleto-infrastructure (V2I) [10]. Ultra reliable low latency communication (URLLC) is required for C-V2X. However, the computing and storage capabilities of vehicle devices are limited, and it is difficult to meet the constraints of a large number of computing requirements and low latency. Therefore, mobile edge computing (MEC) can be introduced into the C-V2X. The C-V2X based on MEC is to move part of the computing power of the core network to the edge of the C-V2X. Generally, the MEC server is equipped with road side unit (RSU). The vehicle uploads the calculation task to the RSU through the V2I communication, and the MEC server corresponding to the RSU calculates the task. When the MEC server completes the calculation, the calculation result is transmitted from the RSU back to the vehicle [11]. And computing requirements and delay constraints can be effectively solved through the combination of MEC and $\mathrm{C}-\mathrm{V} 2 \mathrm{X}$. At the same time, the high-speed mobility of the vehicle makes signal propagation attenuate fast, and the Doppler frequency shift effect is serious. Dynamically changing reflective objects on the road will also deteriorate the channel and affect the received signal strength and quality. In order to solve the abovementioned 
problems, multiple input multiple output (MIMO) technology has been applied in the C-V2X $[12,13]$.

MIMO can suppress channel fading, with two modes of spatial multiplexing and spatial diversity $[14,15]$. The spatial diversity can provide reliable communication, and MIMO can obtain diversity gain by using precoding at the transmitter. Precoding is divided into linear precoding and nonlinear precoding. Compared with linear precoding, nonlinear precoding has higher spectrum utilization. Tomlinson-Harashima precoding (THP) is a typical nonlinear precoding algorithm, which can make a good tradeoff between complexity and performance [16].

When the vehicle is moving at high-speed, the channel state information (CSI) changes significantly during a symbol transmission period. Therefore, traditional channel estimation is not accurate. The fast time-varying characteristics of the channel cause the feedback CSI cannot accurately describe the real-time channel $[17,18]$.

Moreover, if the transmitter adopts the unreasonable transmission power allocation algorithm, it will not be able to obtain communication quality of service (QoS) assurance and better spectrum utilization when the channel has a fast time-varying characteristic [19]. Therefore, the joint power allocation algorithms in the precoding design can effectively improve the transmission reliability and spectrum utilization of the system [20].

Precoding design in high-speed mobile scene has become a research hotspot currently. Zhang et al. [21] proposes a simple precoding and detection scheme that implements precoding by dividing modulated data symbols in the frequency domain into groups and inserting multiple redundant carriers on both sides of each data group. The simulation results show that the scheme can maintain good performance in high-speed scenes, but it does not analyze the influence of the scheme on spectrum utilization. Benmimoune and Massicotte [22] presented a precoding method based on Kerdock code. The method is based on codebook with low complexity, but its overall performance is poor. It can be seen that precoding in high-speed mobile scenes still faces challenges.

Based on the research and analysis, an improved THP algorithm in high-speed mobility scenarios is proposed. The algorithm combines the statistical information with the space-time correlation characteristics of the MIMO channel to construct a dynamic CSI channel model. At the same time, the iterative water filling power allocation algorithm and the multiuser scheduling algorithm based on the greedy algorithm are jointly designed with THP, which can effectively improve the system transmission reliability and spectrum utilization. The main contributions of this paper are as follows:

(1) In order to suppress the channel fading caused by high-speed vehicle movement, we combine MIMO and IoV to construct a MIMO-V2I communication system. Moreover, since the channel in the highspeed mobile environment of vehicles has fast timevarying characteristics, we adopt the dynamic CSI model that combines the statistical information with the space-time correlation characteristics of the channel, which can reflect the current channel characteristics accurately.

(2) Based on the proposed dynamic CSI model, in order to obtain the diversity gain of the MIMO system, an improved THP algorithm is proposed. In the highspeed vehicle moving environment, the algorithm applies the iterative water filling power allocation and the user scheduling based on the greedy algorithm to the THP algorithm for joint optimization design. Firstly, the user scheduling algorithm aims to maximize spectrum utilization and selects the optimal user set according to the channel information of the dynamic CSI model. Secondly, according to the user scheduling result, the channel matrix based on the dynamic CSI model is selected and reconstructed. And the reconstructed channel matrix is applied to the THP algorithm, which can effectively eliminate multiuser interference. The iterative water filling algorithm allocates power to each user according to the channel characteristics of each user in the system, realizing the redistribution of total power, which can effectively improve the transmission efficiency and maximizing the spectrum utilization of the system.

The rest of this paper is organized as follows. Section 2 is the system model. Section 3 proposes an improved THP based on the iterative water filling algorithm in high-speed mobility scenarios, which contains a dynamic CSI model and user scheduling algorithm based on greedy and iterative water filling. Section 4 is the complexity analysis and simulation results of the algorithm. Finally, the conclusion is discussed in Section 5.

\section{System Model}

Assuming that in a multiuser MIMO-IoV system, the infrastructure has deployed $N_{T}$ transmission antennas. For simplicity, it is assumed that infrastructure and $N$ vehicles transmit data, and each vehicle is equipped with an antenna $N_{R}=1$. The $N$ vehicles data are represented by an $N$-dimensional vector $\mathbf{a}=\left[a_{1}, a_{2}, \ldots, a_{N}\right]^{T}$ and are taken from an $M$-QAM constellation with energy $\sigma_{a}^{2}=2(M-1) / 3$.

The multiuser THP system model is shown in Figure 1. It consists of a feedback matrix $\mathbf{B}, N$ nonlinear operators $\bmod _{\sqrt{M}}(x)$, and a feedforward matrix $\mathbf{F}$ with dimensions $N_{T} \times N$. The transmitting signal enters the high-speed channel after the precoding and reaches the receiver. For system physics to achieve, $\mathbf{B}$ is strictly lower triangular to allow data precoding recursively while the modulo operator acts independently over the real and imaginary parts of its input according to the following rule:

$$
\bmod _{\sqrt{M}}(x)=x-2 \sqrt{M} \cdot\left\lfloor\frac{x-\sqrt{M}}{2 \sqrt{M}}\right\rfloor,
$$

where $\lfloor c\rfloor$ indicates the smallest integer larger than or equal to $c$. In practice, $\bmod _{\sqrt{M}}(x)$ performs a periodic mapping of 


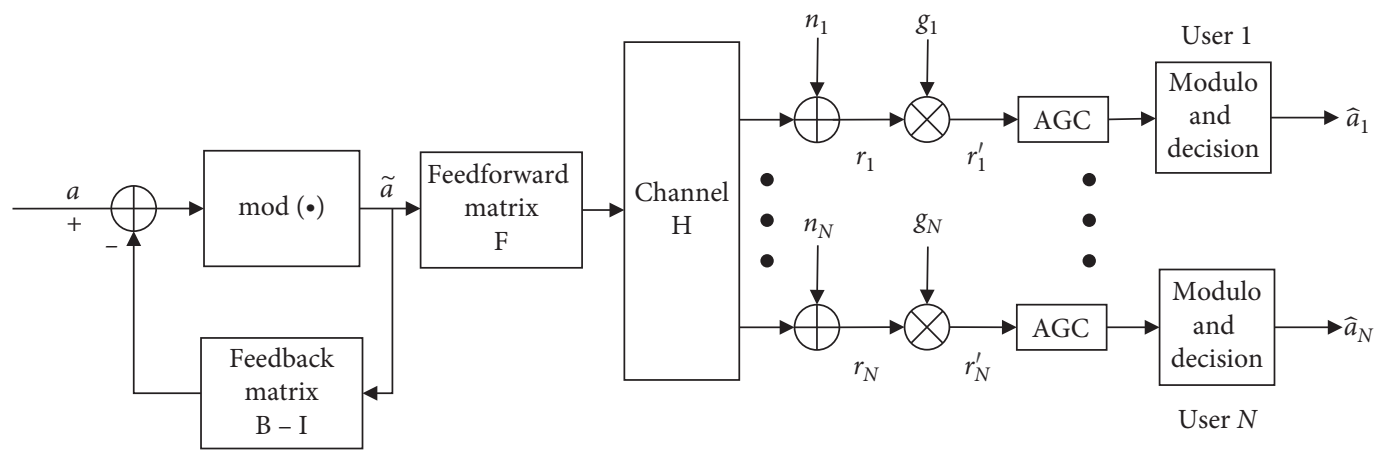

Figure 1: Multiuser THP system model.

the real quantity $x$ onto the interval $(-\sqrt{M}, \sqrt{M}]$. In this way, the precoding symbols $\widetilde{a}_{n}$ are constrained into the square region $\mathcal{N}=\left\{x^{(R)}+j x^{(I)} \mid x^{(R)}, x^{(I)} \in(-\sqrt{M}, \sqrt{M}]\right\}$ and the transmit power is correspondingly reduced with respect to linear prefiltering.

According to (1), we see that the precoding symbols $\tilde{a}_{n}$ can be iteratively computed as follows:

$$
\tilde{a}_{n}=a_{n}-\sum_{\ell=1}^{n-1}[\mathbf{B}-\mathbf{I}]_{n, \ell} \tilde{a}_{l}+d_{n}, \quad n=1,2, \ldots, N,
$$

where $d_{n}=2 \sqrt{M} q_{n}$ and $q_{n}$ is a complex-valued quantity whose real and imaginary parts are suitable integers that reduce $\widetilde{a}_{n}$ to the square region $\aleph$. Equation (2) indicates that the modulo operator in Figure 1 is equivalent to adding a vector $\mathbf{d}=\left[d_{1}, d_{2}, \ldots, d_{N}\right]^{T}$ to the input data symbols. Since the feedback matrix $\mathbf{B}$ is set to strictly lower triangular, we have defined the modified data vector $\mathbf{v}=\mathbf{a}+\mathbf{b}$. Accordingly, the precoding can be rewritten as

$$
\widetilde{\mathbf{a}}=\mathbf{v}-(\mathbf{B}-\mathbf{I}) \widetilde{a} \Longrightarrow \widetilde{\mathbf{a}}=\mathbf{B}^{-1} \mathbf{v} .
$$

The precoding symbols $\widetilde{a}_{n}$ are fed to the forward matrix F. The resulting $N_{T}$-dimensional vector $\mathbf{x}=\mathbf{F} \tilde{a}$ is finally transmitted over the channel using the $N_{T}$ antennas of the infrastructure. The channel is mathematically described by a $N \times N_{T}$ dimensional matrix $\mathbf{H}$. In particular, $[\mathbf{H}]_{n, i}$ represents the channel gain from the $i$-th transmit antenna to the $n$-th receive antenna. The discrete-time signal at the $n$-th mobile vehicle can be written as

$$
r_{n}=\mathbf{h}_{n} \mathbf{F} \tilde{a}+n_{n},
$$

where $\mathbf{h}_{n}$ indicates the $n$-th row of $\mathbf{H}$ while $n_{n}$ is additive white Gaussian noise with zero-mean and variance $\sigma_{n}^{2}$. Stacking the received signals of all users into a single vector $\mathbf{r}=\left[r_{1}, r_{2}, \ldots, r_{N}\right]^{T}$ and submitting in (3), it can be expressed as

$$
\mathbf{r}=\mathbf{H F} \widetilde{a}+\mathbf{n},
$$

where $\mathbf{n}=\left[n_{1}, n_{2}, \ldots, n_{N}\right]^{T}$ is a Gaussian vector with zeromean and covariance matrix $\sigma_{n}^{2} \mathbf{I}$. Since the modulo and the feedback operation are performed at the transmitter, it is equivalent to adding a vector $\mathbf{d}$. At the receiver, each sample $r_{n}$ is passed to the automatic gain control (AGC) and to the same modulo operator employed at the transmitter so as to remove the effect of $d_{n}$. Finally, the received vector can be written as

$$
\mathbf{r}^{\prime}=\mathbf{G}(\mathbf{H F} \tilde{a}+\mathbf{n})=\mathbf{G H F B}^{-1} \mathbf{v}+\mathbf{G n} .
$$

\section{Improved THP Based on Iterative Water Filling Algorithm}

3.1. Dynamic CSI Based on Statistical Information. In the high-speed mobility scenarios, the channel response $h$ of the wireless channel has the characteristics of time-domain selective fading and fast time-varying under the combined influence of multipath effect and Doppler effect [23]. The channel is modeled according to the geometric model. Considering one scattering, the channel impulse response (CIR) expression of the downlink of the MIMO system can be obtained as [23]

$$
h_{p q}(t)=h_{p q}^{\mathrm{LOS}}(t)+h_{p q}^{\mathrm{SB}}(t),
$$

where $h_{p q}^{\mathrm{LOS}}$ and $h_{p q}^{\mathrm{SB}}$ denote the line-of-sight (LOS) and single-bounced (SB) component of the $p$-th transmit antenna to the $q$-th receive antenna, respectively.

$$
\begin{aligned}
h_{p q}^{\mathrm{LOS}}(t) & =C e^{-j 2 \pi f_{c} \tau_{p q}(t)} e^{-j 2 \pi f_{\max } t \cos \left(\phi_{T_{p}}^{\mathrm{LOS}}-\gamma_{R}\right),} \\
h_{p q}^{\mathrm{SB}}(t) & =C \lim _{N \longrightarrow \infty} \sum_{n=1}^{N} \frac{1}{\sqrt{N}} e^{-j\left(\psi_{n}-2 \pi f_{c} \tau_{p q, n}(t)\right)} e^{j 2 \pi f_{\max } t \cos \left(\phi_{R}^{n}(t)-\gamma_{R}\right)},
\end{aligned}
$$

where $C=\sqrt{\left(K_{p q} / K_{p q}+1\right)}, K$ is the Rice factor, $\tau$ denotes the delay on the link, and the link delays on different paths are different, which will result in multipath effects. $e^{-j 2 \pi f_{\max } t \cos \left(\phi_{T_{P}}^{\mathrm{LOS}}-\gamma_{R}\right)}$ and $e^{j 2 \pi f_{\max } t \cos \left(\phi_{R}^{n}(t)-\gamma_{R}\right)}$ denote the Doppler shift. $f_{\max }$ is the maximum Doppler shift of the channel, which is determined by the moving speed of the vehicle. Due to the different angles of arrival (AoA) of the beams on different paths, a Doppler spread effect will be produced, resulting in time-domain selective fading. The coherence time is inversely proportional to the Doppler spread. The larger the Doppler shift, the shorter the coherence time. When the coherence time is less than a symbol transmission period, the current transmission signal 
experiences fast fading. When the channel has a fast timevarying characteristic, the CSI changes significantly. According to the above channel model, the channel matrix can be expressed as

$$
\mathbf{H}=\left[\begin{array}{ccc}
h_{11} & \cdots & h_{1 N_{T}} \\
\vdots & \ddots & \vdots \\
h_{N_{R} 1} & \cdots & h_{N_{R} N_{T}}
\end{array}\right]
$$

Since the CSI is dynamically changed, the channel matrix $\mathbf{H}$ in (9) is corrected by using the statistical characteristics of the channel. In order to distinguish, we use $\mathbf{H}^{D}$ to denote the corrected channel. The channel $\mathbf{H}^{D}$ can be decomposed as into fixed and variable parts as [24]

$$
\mathbf{H}^{D}=\mathbf{H}_{m}+\overline{\mathbf{H}}
$$

where $\mathbf{H}_{m}$ is the channel mean and $\bar{H}$ is the changing part of the channel, which consists of the channel instantaneous value and the correlation value. The mean and instantaneous measurements of the channel constitute the estimated value of the channel $\hat{H}_{s}$. The correlation value can be represented by the error covariance of the channel $\mathbf{R}_{e}$. Thus, at the $s$ moment of transmission time, the channel matrix $\mathbf{H}_{s}$ can be composed of the channel's estimated value $\widehat{H}_{s}$ and its error covariance $\mathbf{R}_{e}$, which is expressed as

$$
\mathbf{H}_{s}=\widehat{H}_{s}+\mathbf{R}_{e}^{(1 / 2)} \text {. }
$$

Assuming that the transmitter has an initial channel measurement $\mathbf{H}_{0}$ at time 0 , together with the channel mean $\mathbf{H}_{m}$, the channel covariance $\mathbf{R}_{0}$ which represent the spatial correlation between all pairs of transmit and receive antennas in the MIMO system, and the autocovariance of the channel $\mathbf{R}_{s}$. Based on minimum mean squared error (MMSE) estimation theory, an optimal estimate $\widehat{h}_{s}$ of the channel at time $s$ and the estimation error covariance $\mathbf{R}_{e, s}$ can be established as

$$
\begin{aligned}
\widehat{h}_{s} & =\mathrm{E}\left[\mathbf{h}_{s} \mid \mathbf{h}_{0}\right]=\mathbf{h}_{m}+\mathbf{R}_{s}^{*} \mathbf{R}_{0}^{-1}\left[\mathbf{h}_{0}-\mathbf{h}_{m}\right], \\
\mathbf{R}_{e, s} & =\operatorname{cov}\left[\mathbf{h}_{s} \mid \mathbf{h}_{0}\right]=\mathbf{R}_{0}-\mathbf{R}_{s}^{*} \mathbf{R}_{0}^{-1} \mathbf{R}_{s},
\end{aligned}
$$

where $\widehat{h}_{s}=\operatorname{vec}\left(\widehat{H}_{s}\right)$. Based on the premise that the channel temporal statistics can be the same for all antenna pairs, it may be assumed that the temporal correlation is homogeneous and identical for any channel element $[25,26]$. The assumption has been used to construct a channel model and verify its existence by measuring the data. Then, the spatial and temporal correlation effects are separable, and the channel autocovariance can be expressed as

$$
\mathbf{R}_{s}=\beta_{s} \mathbf{R}_{0},
$$

where $\beta_{s}=J_{0}\left(2 \pi f_{\max } T_{s}\right)$ is the temporal correlation of channel, which can be obtained according to the Jakes fading model $[27,28] . J_{0}(\cdot)$ denotes the first-kind zero-order Bessel function and $T_{s}$ is the channel sampling time interval. According to (13), we know that all the $N N_{R} \times N_{T}$ channels between the $N_{T}$ transmit and $N N_{R}$ receive antennas have the same temporal correlation function.

Then, we apply the simplified temporal correlation model (12). This model helps to isolate the effect of temporal channel variation on the CSI. The channel estimate $\widehat{H}_{s}$ and its error covariance $\mathbf{R}_{e}$ are rewritten as

$$
\begin{aligned}
\widehat{H}_{s} & =\beta_{s} \mathbf{H}_{0}+\left(1-\beta_{s}\right) \mathbf{H}_{m}, \\
\mathbf{R}_{e} & =\left(1-\beta_{s}^{2}\right) \mathbf{R}_{0},
\end{aligned}
$$

where $\mathbf{H}_{m}=(1 / L) \sum_{j=-L+1}^{0} \mathbf{H}_{j}, L$ is the window length of the CSI mean, and $\mathbf{H}_{j}$ is the instantaneous channel measurement at $j$ sampling time. Then, the transmitter CSI can be briefly characterized as a function of the temporal correlation coefficient of the channel $\beta_{s}$, the measured value of the channel $\mathbf{H}_{0}$, the mean of the channel $\mathbf{H}_{m}$, and covariance of the channel $\mathbf{R}_{0}$.

$\mathbf{R}_{0}$ is a semidefinite Hermitian matrix. Its diagonal elements represent the power gain of the $N N_{R} \times N_{T}$ channels, and the off-diagonal elements are the cross-coupling between these scalar channels. In the channel model constructed based on the Kronecker structure, $\mathbf{R}_{0}$ can be decomposed into the Kronecker product of the transmitter antenna correlation $\mathbf{R}_{t}^{T}$ and the receiver antenna correlation $\mathbf{R}_{r}$ :

$$
\mathbf{R}_{0}=\mathbf{R}_{t}^{T} \otimes \mathbf{R}_{r},
$$

where $\mathbf{R}_{t}$ and $\mathbf{R}_{r}$ both are semidefinite Hermitian matrix. $\mathbf{R}_{t}=\left(1 / N N_{R}\right)\left((1 / L) \sum_{j=-L+1}^{0} \mathbf{H}_{j}^{*} \mathbf{H}_{j}-\mathbf{H}_{m}^{*} \mathbf{H}_{m}\right) \quad$ and $\mathbf{R}_{r}=\left(1 / N_{T}\right)\left((1 / L) \sum_{j=-L+1}^{0} \mathbf{H}_{j}^{*} \mathbf{H}_{j}-\mathbf{H}_{m}^{*} \mathbf{H}_{m}\right)$. Substituting (14) and (15) into (11), the channel matrix $\mathbf{H}^{D}$ based on dynamic CSI can be obtained:

$$
\mathbf{H}^{D}=\beta_{s} \mathbf{H}_{0}+\left(1-\beta_{s}\right) \mathbf{H}_{m}+\left[\left(1-\beta_{s}^{2}\right) \mathbf{R}_{t}^{T} \otimes \mathbf{R}_{r}\right]^{(1 / 2)} .
$$

\subsection{Greedy Algorithm Based on Maximizing Spectrum} Utilization. This paper uses greedy algorithms to select and rank users to maximize spectrum utilization while reducing system bit error ratio (BER). Firstly, a user with the largest spectrum utilization is selected from all $N$ users. Then, we select the next user. Each time the newly selected user and the previously selected user are combined to get the largest spectrum utilization. When the number of selected users reaches the upper limit we set, the selection is stopped and the algorithm ends. The specific process of the algorithm is shown in Algorithm 1.

The greedy algorithm used in this section determines whether to select the joint channel composed of each user and the selected user by continuously calculating whether it has the maximum spectrum utilization. Compared with the traversal algorithm, the search numbers are greatly reduced, and the result is equal to or close to the optimal solution. When the greedy algorithm selects a user, the joint channel matrix is generated, which means the user is synchronously sorted. It can improve the system performance to a certain extent. 
Input: number of transmitting antennas $N_{T}$, signal-to-noise ratio (SNR) $P / N_{0}$, number of candidate users $N$, candidate user channel matrix $\mathbf{H}^{D}$, number of target users $K$;

Output: channel matrix of selected users $\mathbf{H}_{K}$.

Step 1. Initialization: Set candidate user set $\Gamma=\{1,2, \ldots, N\}$, selected user set $\Omega=\varnothing$, channel matrix $\mathbf{H}_{K}=$, which is an empty matrix; Step 2. Calculate the spectrum utilization of each user in the candidate user, and select the user with the largest spectrum utilization. $s_{1}=\operatorname{argmax}_{k_{1} \in \Gamma} \log _{2} \operatorname{det}\left(\mathbf{I}+\left(P / N_{T} N_{0}\right) \mathbf{H}_{k 1} \mathbf{H}_{k 1}^{H}\right)$ bps $/ \mathrm{Hz}$

Step 3. Update the sets. $\Gamma=\Gamma-\left\{s_{1}\right\}, \Omega=\Omega+\left\{s_{1}\right\}$, and update the channel matrix $\mathbf{H}_{K}=\left[\mathbf{H}_{k 1}\right]$;

Step 4. The users in the updated set $\Gamma$ are combined with the selected users to form a new joint channel matrix. Then, calculate the spectrum utilization of the joint channel, and select the user with the largest combined spectrum utilization;

Step 5. Repeat step 3 until the number of target users is selected;

Step 6. End and output the channel matrix of the selected users $\mathbf{H}_{K}$.

Algorithm 1: User scheduling based on the greedy algorithm.

Input: system total power $P$, user number $K$, the weighting matrix $\mathbf{G}$, the feedforward matrix $\mathbf{F}$, the channel matrix $\mathbf{H}_{K}$, threshold $\varepsilon$, water filling line $\mu$;

Output: power allocation matrix for each user $\mathbf{P}_{K}$.

Step 1. Initialize the power distribution, $P_{j}^{(0)}=(P / K), \quad j=1, \ldots, K$;

Step 2. Update power $P_{j}^{(m)}=\left(\mu_{j}^{m}-\left(\sum_{i=1, i \neq j}^{K}\left\|g_{j} \mathbf{h}_{j} \mathbf{f}_{j}\right\|_{F}^{2} P_{j}+n_{R_{j}}\left\|g_{j}\right\|_{F}^{2} \sigma^{2} /\left\|g_{j} \mathbf{h}_{j} \mathbf{f}_{j}\right\|_{F}^{2}\right)\right) j=1, \ldots, K, \sum_{j=1}^{K} P_{j}^{(m-1)}=P$;

Step 3. If $\left(\left|P_{j}^{(m)}-P_{j}^{(m-1)}\right| / P_{j}^{(m)}\right) \leq \varepsilon, j=1, \ldots K$, go to step 4 , or go back to step 2 ;

Step 4. Iterated over, output user power allocation matrix $\mathbf{P}_{K}=\operatorname{diag}\left(P_{1}^{(m)}, P_{2}^{(m)}, \ldots, P_{K}^{(m)}\right)$;

Algorithm 2: Iterative water filling power allocation algorithm.

\subsection{Iterative Water Filling Power Allocation Algorithm.}

The water filling algorithm is a classic power allocation algorithm [29]. The algorithm allocates power to each user based on the channel characteristics to improve the spectrum utilization of the system.

Figure 2 is a diagram of power allocation for a multiuser MIMO precoding system. $P_{j}(j=1, \ldots, K)$ represent the power allocated to each user, $\mathbf{h}_{j}$ represents the $j$-th row of the channel matrix $\mathbf{H}_{K}$, and $\mathbf{f}_{j}$ represents the $j$-th column of the feedforward matrix $\mathbf{F}$, so the spectrum utilization can be expressed as

$$
C=\sum_{j=1}^{K} \log _{2}\left(1+\frac{\left\|g_{j} \mathbf{h}_{j} \mathbf{f}_{j}\right\|_{F}^{2} P_{j}}{\sum_{i=1, i \neq j}^{K}\left\|g_{j} \mathbf{h}_{j} \mathbf{f}_{j}\right\|_{F}^{2} P_{j}+n_{R_{j}}\left\|g_{j}\right\|_{F}^{2} \sigma^{2}}\right) .
$$

To maximize spectrum utilization, we can construct objective function and constrain as follows:

$$
\left\{\begin{array}{l}
\max C=\sum_{j=1}^{K} C_{j}\left(P_{j}\right), \\
\text { s.t. } \sum_{j=1}^{K} P_{j}=P \text { and } P_{j} \geq 0, \quad j=1, \ldots, K .
\end{array}\right.
$$

An iterative water filling algorithm suitable for multiuser MIMO systems is designed. Firstly, the power of each user is initialized, and the power is distributed equally after obtaining the precoding matrix. Then, the user power is iteratively updated according to the objective function. In

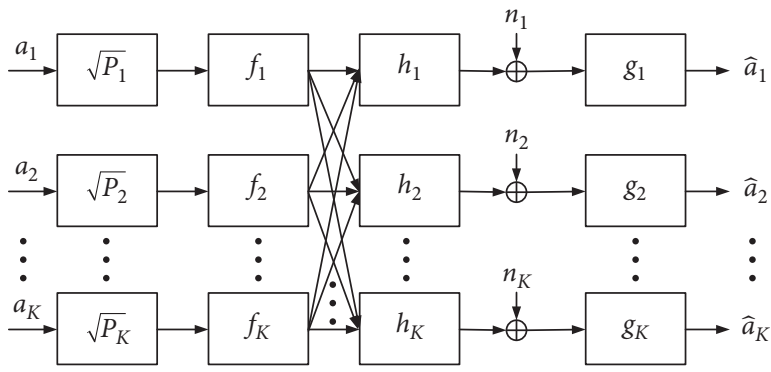

FIGURE 2: Diagram of power allocation for the multiuser precoding system.

each iteration, the interference is equivalent to noise and processed, and the user power allocation is optimized through the water filling algorithm. When the power allocated by each user basically does not change, the iteration stops and the algorithm ends. The specific process of the iterative water filling power allocation algorithm is as follows.

The iterative water filling algorithm takes full account of the influence of interference and treats it as noise. When there is no spatial correlation in the channel, the singular value distribution of each user channel is relatively average, and the system using the iterative water filling algorithm does not have significant channel capacity. If the channel correlation exists, the channel condition number is large, or even the channel rank is not satisfied, the channel capacity advantage of the iterative water filling algorithm will be more obvious. 


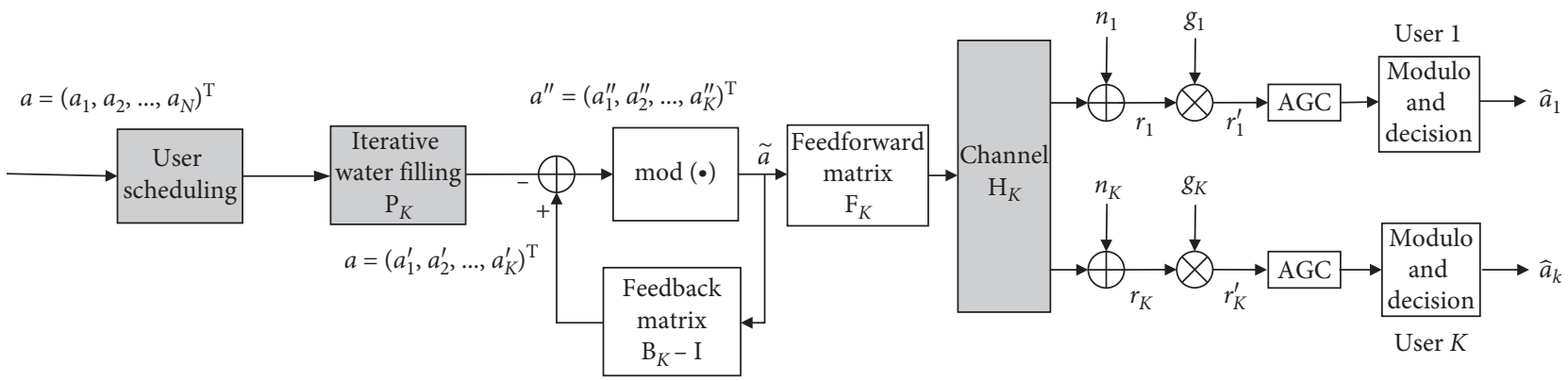

FIgURE 3: Diagram of improved THP based on the iterative water filling algorithm.

3.4. Precoding Design. The diagram of improved THP based on the iterative water filling algorithm is shown in Figure 3. The original transmission signal is $\mathbf{a}=\left[a_{1}, a_{2}, \ldots, a_{N}\right]^{T}$. The transmission signal after user scheduling and sorting by the greedy algorithm proposed in subsection $\mathscr{B}$ is $a^{\prime}=\left[a_{1}^{\prime}, a_{2}^{\prime}, \ldots, a_{K}^{\prime}\right]^{T}$, and the transmission signal after power allocation by the iterative water injection algorithm proposed in subsection $\mathscr{C}$ is $a^{\prime \prime}=\left[a_{1}^{\prime \prime}, a_{2}^{\prime \prime}, \ldots, a_{K}^{\prime \prime}\right]^{T}$. After the user scheduling, reconstructed channel matrix $\mathbf{H}_{K}$ will generate the corresponding feedback matrix $\mathbf{B}_{K}$, a weighting matrix $\mathbf{G}_{K}$, and a feedforward matrix $\mathbf{F}_{K}$. Then, the received vector $\mathbf{r}_{K}^{\prime}$ is

$$
\mathbf{r}_{K}^{\prime}=\mathbf{G}_{K}\left(\mathbf{H}_{K} \mathbf{F}_{K} \tilde{a}+\mathbf{n}_{K}\right)=\mathbf{G}_{K} \mathbf{H}_{K} \mathbf{F}_{K} \mathbf{B}_{K}^{-1} \mathbf{v}_{K}+\mathbf{G}_{K} \mathbf{n}_{K},
$$

where $\mathbf{r}_{K}^{\prime}=\left[r_{1}^{\prime}, r_{2}^{\prime}, \ldots, r_{K}^{\prime}\right]^{T}, \quad \mathbf{n}_{K}^{\prime}=\left[n_{1}, n_{2}, \ldots, n_{K}\right]^{T}$, and $\mathbf{v}_{K}^{\prime}=\mathbf{B}_{K}^{\prime} \tilde{a}$ are the equivalent transmit data vector. As can be seen from Figure 3, the error e between the received vector $\mathbf{r}_{K}^{\prime}$ and the equivalent transmitted vector $\mathbf{v}_{K}$ is expressed as

$$
\begin{aligned}
\mathbf{e} & =\mathbf{r}_{K}^{\prime}-\mathbf{v}_{K}^{\prime} \\
& =\mathbf{G}_{K}\left(\mathbf{H}_{K} \mathbf{F}_{K} \widetilde{a}+\mathbf{n}_{K}\right)-\mathbf{v}_{K} \\
& =\left(\mathbf{G}_{K} \mathbf{H}_{K} \mathbf{F}_{K}-\mathbf{B}_{K}\right) \widetilde{a}+\mathbf{G}_{K} \mathbf{n}_{K} .
\end{aligned}
$$

According to the MMSE algorithm, we can construct objective functions and constraints as follows:

$$
\left\{\begin{array}{l}
\underset{\mathbf{B}_{K}, \mathbf{G}_{K}, \mathbf{F}_{K}}{\arg \min } E\left[\left\|\left(\mathbf{G}_{K} \mathbf{H}_{K} \mathbf{F}_{K}-\mathbf{B}_{K}\right) \tilde{a}+\mathbf{G}_{K} n_{K}\right\|^{2}\right], \\
\text { s.t. }\|\tilde{a}\|^{2} \leq P,
\end{array}\right.
$$

where $P$ represents the total transmit power at the transmitter. By using the orthogonal principle, we have

$$
\begin{aligned}
E\left[\mathbf{e r}_{K}^{H}\right] & =E\left[\left(\mathbf{r}_{K}^{\prime}-\mathbf{v}_{K}\right) \mathbf{r}_{K}^{H}\right] \\
& =E\left[\mathbf{G}_{K} \mathbf{r}_{K} \mathbf{r}_{K}^{H}-\mathbf{B}_{K} \widetilde{a} \mathbf{r}_{K}^{H}\right] \\
& =0 .
\end{aligned}
$$

Substituting (20) into (22), we have

$$
\mathbf{G}_{K}\left(\mathbf{H}_{K} \mathbf{F}_{K} \mathbf{F}_{K}^{H} \mathbf{H}_{K}^{H}+\xi \mathbf{I}_{K}\right)=\mathbf{B}_{K} \mathbf{F}_{K}^{H} \mathbf{H}_{K}^{H},
$$

where $\xi=\left(\sigma_{\mathbf{n}_{K}}^{2} / \sigma_{\widetilde{a}}^{2}\right)=\left(E\left[\mathbf{n}_{K} \mathbf{n}_{K}^{H}\right] / E\left[\widetilde{a} \widetilde{a}^{H}\right]\right)$. Assuming that the feedforward matrix $\mathbf{F}_{K}$ is a unitary matrix which satisfies $\mathbf{F}_{K} \mathbf{F}_{K}^{H}=\mathbf{I}$, then we have

$$
\mathbf{G}_{K}^{-1} \mathbf{B}_{K} \mathbf{B}_{K}^{H} \mathbf{G}_{K}^{-H}=\left(\mathbf{H}_{K} \mathbf{H}_{K}^{H}+\xi \mathbf{I}\right) \mathbf{H}_{K}^{-H} \mathbf{H}_{K}^{-1}\left(\mathbf{H}_{K} \mathbf{H}_{K}^{H}+\xi \mathbf{I}\right)^{H}
$$

Let $\mathbf{L}_{K}=\mathbf{G}_{K}^{-1} \mathbf{B}_{K}$, then (25) can be expressed as

$$
\mathbf{L}_{K} \mathbf{L}_{K}^{H}=\left(\mathbf{H}_{K} \mathbf{H}_{K}^{H}+\xi \mathbf{I}\right) \mathbf{H}_{K}^{-H} \mathbf{H}_{K}^{-1}\left(\mathbf{H}_{K} \mathbf{H}_{K}^{H}+\xi \mathbf{I}\right)^{H} .
$$

We perform a LQ decomposition of $\left(\mathbf{H}_{K} \mathbf{H}_{K}^{H}+\xi \mathbf{I}\right) \mathbf{H}_{K}^{-H}$ and obtain the main diagonal element of the lower triangular matrix $\mathbf{L}_{K}$. Then, we can obtain the precoding matrices.

$$
\begin{aligned}
\mathbf{G}_{K} & =\left[\begin{array}{llll}
l_{11}^{-1} & & & \\
& l_{22}^{-1} & & \\
& & \ddots & \\
& & & l_{K K}^{-1}
\end{array}\right], \\
\mathbf{B}_{K} & =\mathbf{G}_{K} \mathbf{L}_{K}, \\
\mathbf{F}_{K} & =\mathbf{Q}_{K} .
\end{aligned}
$$

\section{Complexity and Simulation Analysis}

This section mainly analyzes the complexity of the proposed algorithm and several other classical precoding algorithms. The simulation is performed by Matlab, and finally the simulation results are analyzed.

4.1. Complexity Analysis. We compare the computational complexity of the classical linear precoding algorithm, the THP, and the improved THP algorithm in Table 1. It should be noted that the complexity in this paper mainly refers to the number of times the algorithm performs the product operation once.

The zero force (ZF) and MMSE algorithms in Table 1 are classical linear precoding algorithms. The linear precoding algorithm has lower complexity, but its BER performance is poor and the spectrum utilization is low. Compared with ZF, MMSE has a matrix inverse operation, so its complexity is slightly higher than the ZF. The traditional THP algorithm without user scheduling introduces QR decomposition of matrix, so its complexity is higher than that of linear algorithms. Furthermore, the user-scheduled THP algorithm introduces a user scheduling algorithm based on the greedy algorithms, which is less complex than the traversal search. The THP algorithm with the dynamic CSI model introduces 
TABle 1: Complexity comparison.

\begin{tabular}{lc}
\hline Algorithm & Complexity \\
\hline ZF [30] & $N_{T}^{3}$ \\
MMSE [30] & $4 N_{T}^{3}+N_{T}$ \\
ZF-THP (without user scheduling) [16] & $3 N_{T}^{3}+3 N_{T}^{2}+N_{T}$ \\
MMSE-THP (without user scheduling) [16] & $7 N_{T}^{3}+3 N_{T}^{2}+2 N_{T}$ \\
ZF-THP (with user scheduling) & $3 N_{T}^{3}+3 N_{T}^{2}+N_{T}+K(K+1)(2 K+1)\left(N_{T} / 6\right)$ \\
MMSE-THP (with user scheduling) & $7 N_{T}^{3}+3 N_{T}^{2}+2 N_{T}+K(K+1)(2 K+1)\left(N_{T} / 6\right)$ \\
ZF-THP (dynamic CSI model) & $N_{T}^{4}+3 N_{T}^{3}+3 N_{T}^{2}+3 N_{T}$ \\
MMSE-THP (dynamic CSI model) & $N_{T}^{4}+7 N_{T}^{3}+3 N_{T}^{2}+4 N_{T}$ \\
The proposed algorithm (ZF) & $N_{T}^{4}+3 N_{T}^{3}+3 N_{T}^{2}+3 N_{T}+K(K+1)(2 K+1)\left(N_{T} / 6\right)$ \\
The proposed algorithm (MMSE) & $N_{T}^{4}+7 N_{T}^{3}+3 N_{T}^{2}+4 N_{T}+K(K+1)(2 K+1)\left(N_{T} / 6\right)$ \\
\hline
\end{tabular}

Kronecker product operation with complexity $N_{T}^{4}$, so its complexity is higher than traditional THP algorithm. The proposed algorithm not only adds the dynamic CSI model and user scheduling but also adds the iterative water filling power allocation algorithm, so the complexity is higher than other algorithms. However, from a practical point of view, the computational complexity of the proposed algorithm is still within acceptable limits.

4.2. Simulation Analysis. In order to verify the rationality of the proposed algorithm, we construct a MIMO-V2I communication system to simulate and verify the proposed algorithm in a high-speed mobile scenario. In the simulation, a $4 \times 1$ multiuser MIMO model is adopted. The channel model is WINNER II channel [23]. The simulation system parameters are shown in Table 2.

This paper mainly introduces BER and spectrum utilization of linear precoding [30], the traditional THP algorithm [16], and the proposed algorithm in different speed scenarios.

Figures 4(a) and 4(b) show the BER of linear precoding, traditional THP, and the proposed algorithm when the vehicle speed is $120 \mathrm{~km} / \mathrm{h}$ and $30 \mathrm{~km} / \mathrm{h}$.

In high-speed scenarios, the proposed algorithm is the best. The traditional THP algorithm based on the dynamic CSI model is second, and the traditional THP algorithm combined with the user scheduling algorithm is better than the traditional THP. The linear precoding is the worst. This is because the dynamic CSI model compensates for the instantaneous channel through channel correlation, so the CSI model obtained through feedback is closer to the actual channel, thereby improving system performance. The user scheduling algorithm based on the greedy algorithm preferentially selects the user with the highest spectrum utilization rate for priority transmission, which not only improves the spectrum utilization rate of the system but also effectively filters out noise and interference, thereby reducing BER.

In low-speed scenarios, the BER of various precoding algorithms is consistent with the trend of BER in high-speed scenarios. We observe that the precoding algorithm combined with the dynamic CSI model is close to that of the precoding algorithm without the dynamic CSI model. This is because the moving speed is low, there is no strong
TABLE 2: Simulation parameters.

\begin{tabular}{lc}
\hline Parameters & Value \\
\hline Carrier frequency & $5.9 \mathrm{GHz}$ \\
Moving speed & $30 \mathrm{~km} / \mathrm{h}, 120 \mathrm{~km} / \mathrm{h}$ \\
Transmitting antennas $N_{T}$ & 4 \\
Receiving antennas $N_{R}$ & 1 \\
Total users $N$ & 10 \\
Target users $K$ & 4 \\
Modulation & QPSK \\
\hline
\end{tabular}

correlation between channels, and the BER advantage brought by the dynamic CSI model is not obvious.

In summary, the proposed algorithm is suitable for highspeed mobile scenarios.

Figures 5(a) and 5(b) show the spectrum utilization of linear precoding, traditional THP, and the proposed algorithm at a vehicle speed of $120 \mathrm{~km} / \mathrm{h}$ and $30 \mathrm{~km} / \mathrm{h}$.

In high-speed scenarios, the proposed algorithm has the best spectrum utilization performance. This is because the multiuser scheduling algorithm based on the greedy algorithm optimizes the target by maximizing the channel capacity, thereby improving the spectrum utilization of the system. Moreover, the dynamic CSI model makes the channel closer to the actual channel, and the accurate CSI can design a better precoding matrix and further improve the spectrum utilization.

In low-speed scenarios, the trend of precoding algorithm spectrum utilization is consistent with that in high-speed scenarios. However, in low-speed scenarios, the spectrum utilization of each precoding algorithm is higher than that of similar algorithms in high-speed situations. According to information theory, when the SNR is determined, the spectrum utilization is only related to the channel matrix. In the low-speed scene, the channel is corrected by channel correlation, and the channel gain is improved so that the spectrum utilization is effectively improved to a certain extent.

Figure 6 shows the spectrum utilization comparison between the precoding algorithm using the iterative water filling algorithm and the precoding algorithm using equal power allocation. As illustrated, spectrum utilization is improved by using iterative water filling power allocation. The adopted iterative water filling algorithm carries out adaptive power allocation for users. More power is allocated 

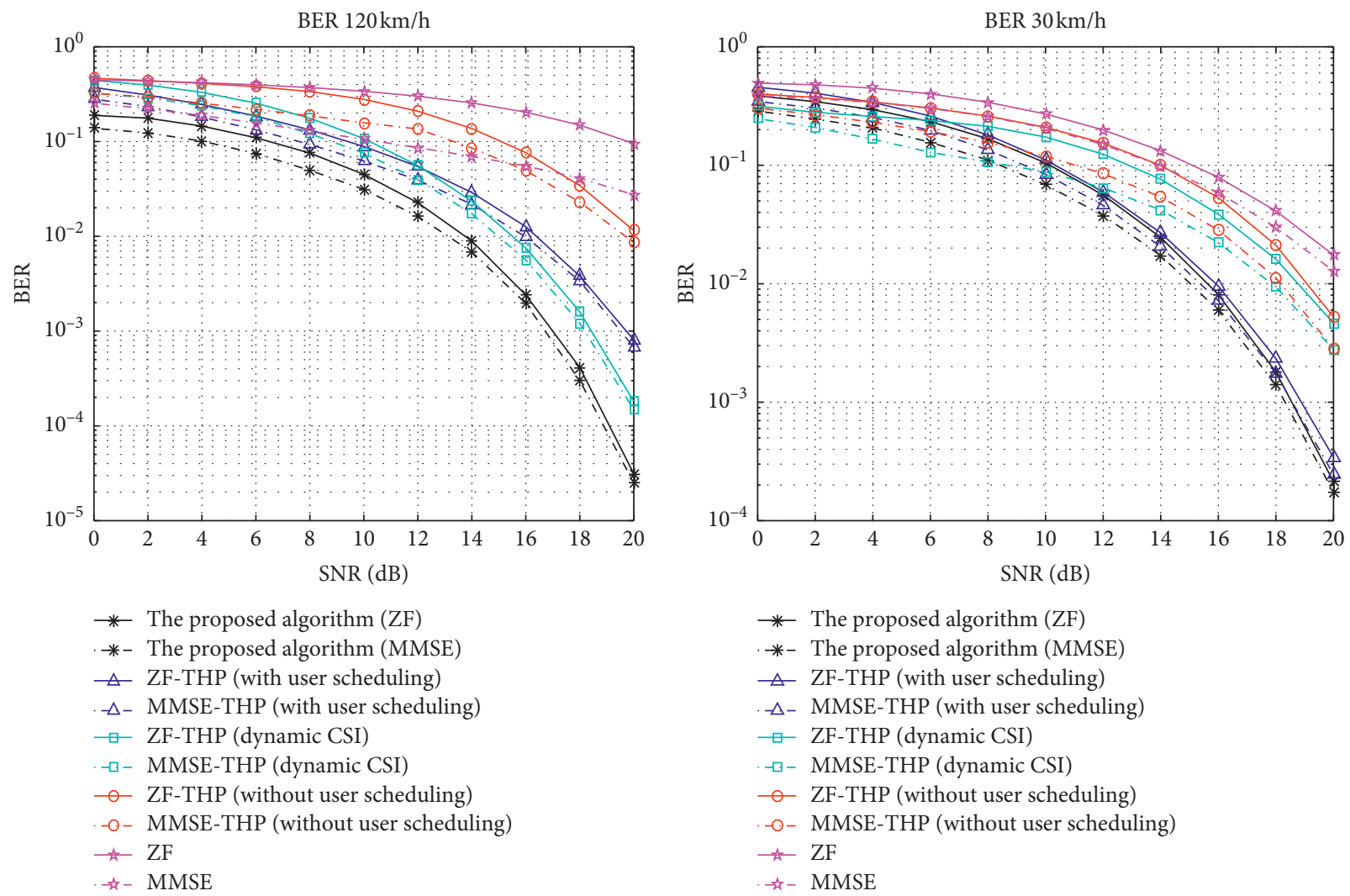

(a)

(b)

FIGURE 4: BER of several precoding algorithms: (a) $120 \mathrm{~km} / \mathrm{h}$; (b) $30 \mathrm{~km} / \mathrm{h}$.
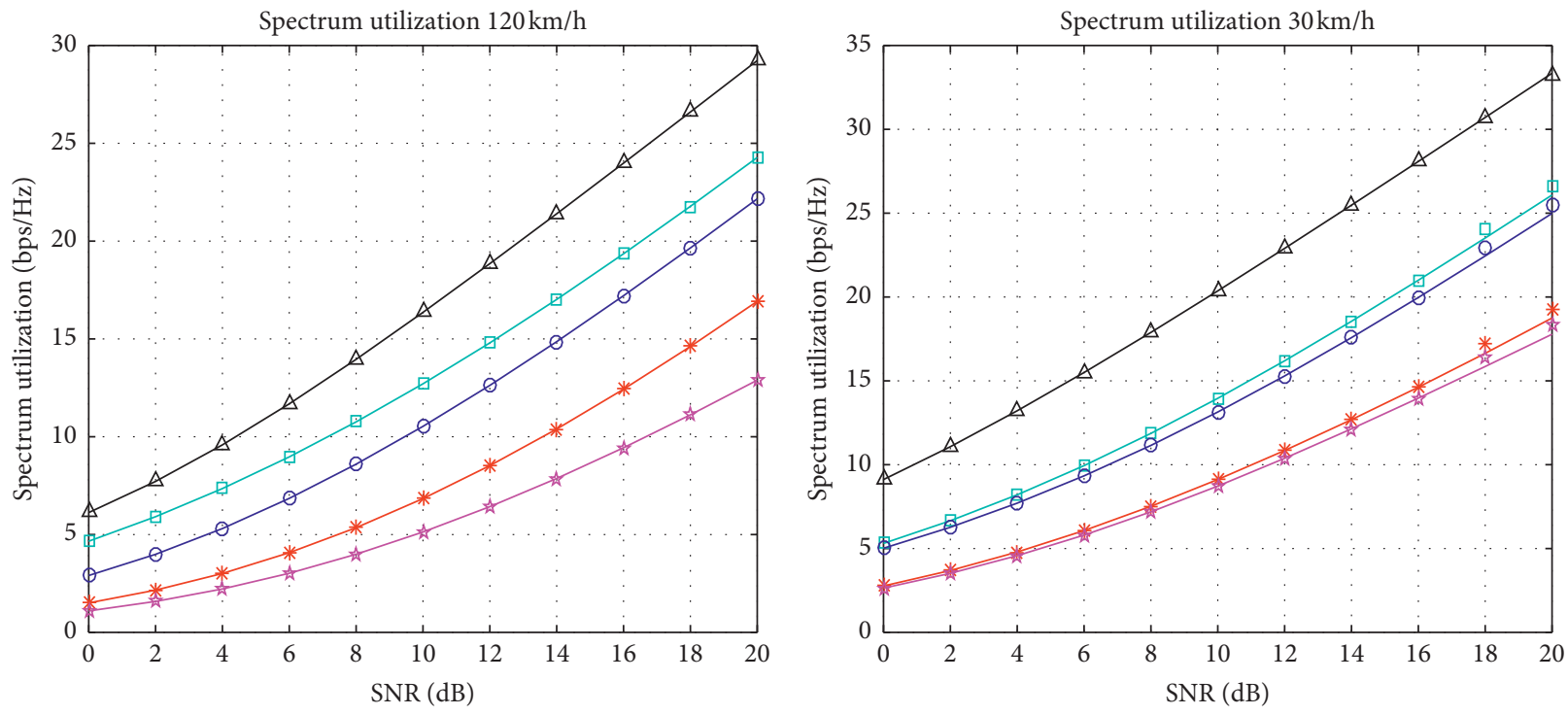

$\triangle$ The proposed algorithm
- THP (with user scheduling)
- THP (dynamic CSI)
* THP (without user scheduling)
- Linear

(a)

$$
\begin{aligned}
& \triangle \text { The proposed algorithm } \\
& -\square \text { THP (with user scheduling) } \\
& - \text { THP (dynamic CSI) } \\
& \text { * THP (without user scheduling) } \\
& \text { * Linear }
\end{aligned}
$$

(b)

FIGURE 5: Spectrum utilization of several precoding algorithms: (a) $120 \mathrm{~km} / \mathrm{h}$; (b) $30 \mathrm{~km} / \mathrm{h}$. 


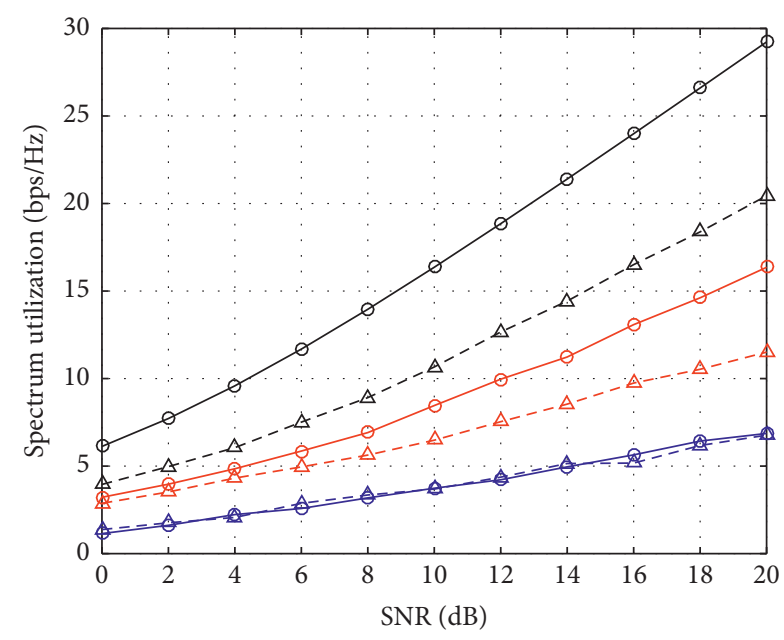

$\multimap N_{T}=N_{R}=1$ (iter water-filling)

- $N_{T}=N_{R}=2$ (iter water-filling)

$\multimap N_{T}=N_{R}=4$ (iter water-filling)

- $\triangle-N_{T}=N_{R}=1$ (average power)

- $\triangle-N_{T}=N_{R}=2$ (average power)

- $\Delta-N_{T}=N_{R}=4$ (average power)

FIGURE 6: Spectrum utilization under different power allocation algorithms.

to users with good channel conditions while less or no power is allocated with poor channel conditions. The power resources are effectively utilized so that the spectrum utilization of the system is improved as a whole. At the same time, with the increase in the antennas number, the spectrum utilization of the iterative water filling power allocation algorithm has obvious advantages. It is verified that the iterative water filling power algorithm is suitable for the MIMO systems with multiple antennas at the transmitter and receiver.

\section{Conclusion}

This paper studies the precoding of the ultra reliable communication for V2I of CPSs. Aiming at the fast time-varying characteristics of the channel in high-speed mobility scenarios, we propose an improved THP algorithm. By constructing a dynamic CSI model based on channel statistic information and related characteristics, the obtained dynamic CSI is closer to the current actual channel. At the same time, the iterative water filling power allocation algorithm and the user scheduling algorithm based on the greedy algorithm are combined with the THP algorithm to carry out joint optimization design. The simulation results show that the proposed algorithm has better BER and spectrum utilization than the traditional THP algorithm and linear precoding algorithm and is more suitable for fast time-varying channel environment in high-speed mobility scenarios.

\section{Data Availability}

The data used to support the findings of this study are included within the article.

\section{Conflicts of Interest}

The authors declare that they have no conflicts of interest regarding the publication of this manuscript.

\section{Acknowledgments}

This work was supported by the National Natural Science Foundation of China (no. 61501066) and Natural Science Foundation of Chongqing (no. cstc2019jcyj-msxmX0017).

\section{References}

[1] Y. Zhou, F. R. Yu, J. Chen, and Y. Kuo, "Cyber-physical-social systems: a state-of-the-art survey, challenges and opportunities," IEEE Communications Surveys \& Tutorials, vol. 22, no. 1, pp. 389-425, 2020.

[2] Q. Sun, Y. Zhang, H. He, D. Ma, and H. Zhang, "A novel energy function-based stability evaluation and nonlinear control approach for energy internet," IEEE Transactions on Smart Grid, vol. 8, no. 3, pp. 1195-1210, 2017.

[3] Q. Sun, S. Chen, L. Chen, and D. Ma, "Quasi-Z-source network-based hybrid power supply system for aluminum electrolysis industry," IEEE Transactions on Industrial Informatics, vol. 13, no. 3, pp. 1141-1151, 2017.

[4] L. Zhu, F. R. Yu, Y. Wang, B. Ning, and T. Tang, "Big data analytics in intelligent transportation systems: a survey," IEEE Transactions on Intelligent Transportation Systems, vol. 20, no. 1, pp. 383-398, 2019.

[5] R. Ghosh, R. Pragathi, S. Ullas, and S. Borra, "Intelligent transportation systems: a survey," in Proceedings of the 2017 International Conference on Circuits, Controls, and Communications (CCUBE), pp. 160-165, Bangalore, India, December 2017.

[6] W. Hu, C. Ruan, H. Nian, and D. Sun, "Zero-sequence current suppression strategy with common-mode voltage control for open-end winding PMSM drives with common DC bus," IEEE Transactions on Industrial Electronics, vol. 68, no. 6, pp. 4691-4702, 2021.

[7] W. Rui, S. Qiuye, M. Dazhong, and H. Xuguang, "Line impedance cooperative stability region identification method for grid-tied inverters under weak grids," IEEE Transactions on Smart Grid, vol. 11, no. 4, pp. 2856-2866, 2020.

[8] R. Wang, Q. Sun, W. Hu, Y. Li, D. Ma, and P. Wang, "SoCbased droop coefficients stability region analysis of the battery for stand-alone supply systems with constant power loads," IEEE Transactions on Power Electronics, vol. 36, no. 7, pp. 7866-7879, 2021.

[9] R. Wang, Q. Sun, D. Ma, and Z. Liu, "The small-signal stability analysis of the droop-controlled converter in electromagnetic timescale," IEEE Transactions on Sustainable Energy, vol. 10, no. 3, pp. 1459-1469, 2019.

[10] T. Wang, X. Wang, Z. Cui, Y. Cao, and C. Suthaputchakun, "Survey on cooperatively V2X downloading for intelligent transport systems," IET Intelligent Transport Systems, vol. 13, no. 1, pp. 13-21, 2019.

[11] I. Shaer, A. Haque, and A. Shami, "Multi-component V2X applications placement in edge computing environment," in Proceedings of the 2020 IEEE International Conference on Communications (ICC 2020), pp. 1-6, Dublin, Ireland, June 2020.

[12] S. Moon and I. Hwang, "Design and performance analysis of vehicle MIMO system for NR-based enhanced V2X," in Proceedings of the 2018 International Conference on 
Information and Communication Technology Convergence (ICTC), pp. 351-355, Jeju, Korea (South), October 2018.

[13] B. Wang, R. Shi, C. Ji, and J. Hu, "Joint precoding and user scheduling for full-duplex cooperative MIMO-NOMA V2X networks," in Proceedings of the IEEE Vehicular Technology Conference (VTC2019-Fall), pp. 1-6, Honolulu, HI, USA, September 2019.

[14] Y. Wu, C. Xiao, Z. Ding, X. Gao, and S. Jin, “A survey on MIMO transmission with finite input signals: technical challenges, advances, and future trends," Proceedings of the IEEE, vol. 106, no. 10, pp. 1779-1833, 2018.

[15] E. Castañeda, A. Silva, A. Gameiro, and M. Kountouris, “An overview on resource allocation techniques for multi-user MIMO systems," IEEE Communications Surveys \& Tutorials, vol. 19, no. 1, pp. 239-284, 2017.

[16] X. Guo, D. Yang, H. Wang, J. Kuang, and X. Wen, "MMSETHP with QoS requirements for the downlink of multiuser MIMO systems," in Proceedings of the 2018 88th IEEE Vehicular Technology Conference (VTC-Fall), pp. 1-5, Chicago, IL, USA, August 2018.

[17] M. Vu and A. Paulraj, "On the capacity of MIMO wireless channels with dynamic CSIT," IEEE Journal on Selected Areas in Communications, vol. 25, no. 7, pp. 1269-1283, 2007.

[18] J. Wang, M. Bengtsson, B. Ottersten, and A. P. Palomar, "Robust MIMO precoding for several classes of channel uncertainty," IEEE Transactions on Signal Processing, vol. 61, no. 12, pp. 3056-3070, 2013.

[19] C. Kotchasarn, "Power allocation for multi-user downlink MIMO transmissions," in Proceedings of the IEEE International Conference on Innovative Research and Development (ICIRD), pp. 1-5, Bangkok, Thailand, May 2018.

[20] K. A. Bonsu, W. Zhou, S. Pan, and Y. Yan, "Optimal power allocation with limited feedback of channel state information in multi-user MIMO systems," China Communications, vol. 17, no. 2, pp. 163-175, 2020.

[21] R. Zhang, B. Ai, L. Yang, H. Song, and Z.-Q. Li, “A precoding and detection scheme for OFDM based wireless communication system in high-speed environment," IEEE Transactions on Consumer Electronics, vol. 60, no. 4, pp. 558-566, 2014.

[22] M. Benmimoune and D. Massicotte, "Multi-user MIMO precoding with Kerdock codebook," in Proceedings of the 2010 7th International Symposium on Wireless Communication System, pp. 71-75, York, UK, September 2010.

[23] A. Ghazal, Y. Yuan, C.-X. Wang et al., "A non-stationary IMT-advanced MIMO Channel Model for high-mobility wireless communication systems," IEEE Transactions on Wireless Communications, vol. 16, no. 4, pp. 2057-2068, 2017.

[24] M. Vu and A. Paulraj, "Optimal linear precoders for MIMO wireless correlated channels with nonzero mean in space-time coded systems," IEEE Transactions on Signal Processing, vol. 54, no. 6, pp. 2318-2332, 2006.

[25] Y. Li, X. Cheng, and N. Zhang, "Deterministic and stochastic simulators for non-isotropic V2V-MIMO wideband channels," China Communications, vol. 15, no. 7, pp. 18-29, 2018.

[26] Y. Yuan, C.-X. Wang, Y. He, M. M. Alwakeel, and E.-H. M. Aggoune, "3D wideband non-stationary geometrybased stochastic models for non-isotropic MIMO vehicle-tovehicle channels," IEEE Transactions on Wireless Communications, vol. 14, no. 12, pp. 6883-6895, 2015.

[27] C. Kong, C. Zhong, A. K. Papazafeiropoulos, M. Matthaiou, and Z. Zhang, "Sum-rate and power scaling of massive MIMO systems with channel aging," IEEE Transactions on Communications, vol. 63, no. 12, pp. 4879-4893, 2015.
[28] L. Zhao, K. Zheng, H. Long, H. Zhao, and W. Wang, "Performance analysis for downlink massive multiple-input multiple-output system with channel state information delay under maximum ratio transmission precoding," IET Communications, vol. 8, no. 3, pp. 390-398, 2014.

[29] P. He, L. Zhao, and B. Venkatesh, "Novel water-filling for maximum throughput of power grid, MIMO, and energy harvesting coexisting system with mixed constraints," IEEE Transactions on Communications, vol. 65, no. 2, pp. 827-838, 2017.

[30] T. K. Lyu, "Capacity of multi-user MIMO systems with MMSE and ZF precoding," in Proceedings of the 2016 IEEE Conference on Computer Communications Workshops (INFOCOM WKSHPS), pp. 1083-1084, San Francisco, CA, USA, April 2016. 